\title{
Clonal multidrug-resistant Corynebacterium striatum within a nosocomial environment, Rio de Janeiro, Brazil
}

\author{
Paulo Victor Pereira Baioo, ${ }^{1,4,5}$, Higor Franceschi Mota', Andréa D'avila Freitas ${ }^{1,2}$, \\ Débora Leandro Rama Gomes ${ }^{1,6}$, Juliana Nunes Ramos ${ }^{1,4}$, Lincoln Oliveira Sant'Anna', \\ Mônica Cristina Souza', Thereza Cristina Ferreira Camello, ${ }^{1,3}$, Raphael Hirata Junior ${ }^{1}$, \\ Verônica Viana Vieira ${ }^{4}$, Ana Luíza Mattos-Guaraldi++
}

\author{
'Laboratório de Difteria e Corinebactérias de Importância Clínica, Departamento de Microbiologia, \\ Imunologia e Patologia, Faculdade de Ciências Médicas ${ }^{2}$ Unidade Docente Assistencial de Doenças Infecciosas e Parasitárias \\ ${ }^{3}$ Laboratório de Bacteriologia, Hospital Universitário Pedro Ernesto, Universidade do Estado do Rio de Janeiro, Rio de Janeiro, RJ, Brasil \\ ${ }^{4}$ Departamento de Microbiologia, Instituto Nacional de Controle de Qualidade em Saúde-Fiocruz, Rio de Janeiro, RJ, Brasil \\ ${ }^{5}$ Laboratório Químico Farmacêutico do Exército, Ministério da Defesa, Rio de Janeiro, RJ, Brasil \\ ${ }^{6}$ Faculdade de Farmácia, Instituto Federal de Educação, Ciência e Tecnologia do Rio de Janeiro, Rio de Janeiro, RJ, Brasil
}

Corynebacterium striatum is a potentially pathogenic microorganism with the ability to produce outbreaks of nosocomial infections. Here, we document a nosocomial outbreak caused by multidrug-resistant (MDR) C. striatum in Rio de Janeiro, Brazil. C. striatum identification was confirmed by 16S rRNA and $\mathrm{rpo} B$ gene sequencing. Fifteen C. striatum strains were isolated from adults (half of whom were 50 years of age and older). C. striatum was mostly isolated in pure culture from tracheal aspirates of patients undergoing endotracheal intubation procedures. The analysis by pulsed-field gel electrophoresis (PFGE) indicated the presence of four PFGE profiles, including two related clones of MDR strains (PFGE I and II). The data demonstrated the predominance of PFGE type I, comprising 11 MDR isolates that were mostly isolated from intensive care units and surgical wards. A potential causal link between death and MDR C. striatum (PFGE types I and II) infection was observed in five cases.

Key words: Corynebacterium striatum - nosocomial outbreak - PFGE - multidrug-resistant clones

Like several other multidrug-resistant (MDR) nondiphtherial Corynebacterium species, Corynebacterium striatum has been cited with increased frequency as a pathogen of nosocomial infections (Lee et al. 2005), including septicaemias (Martín et al. 2003), pulmonary infection (Renom et al. 2007, Wong et al. 2010), meningitis (Weiss et al. 1996), endocarditis (Oliva et al. 2010), osteomyelitis (Fernández-Ayala et al. 2001), septic arthritis (Scholle 2007), keratitis (Heidemann et al. 1991), skin wounds (Moore et al. 2010) and intrauterine infections (Boltin et al. 2009). The first case of C. striatum in an uncomplicated urinary tract infection in an ambulatory patient without any other predisposing factors was recently reported (López et al. 2009).

Outbreaks caused by C. striatum have been reported in long-term hospitalised patients with prolonged exposure to broad-spectrum antibiotics and in intensive care units (ICU) (Leonard et al. 1994, Otsuka et al. 2006). The presence of multiple medical devices may facilitate

Financial support: CAPES, FAPERJ, CNPq, PAPES V-FIOCRUZ/ CNPq, SR-2/UERJ, PNPD-CAPES/MEC, PAPD-FAPERJ/CAPES PVPB and HFM contributed equally to this work.

+ Corresponding author: guaraldi@uerj.br

Received 13 February 2012

Accepted 2 October 2012 colonisation by $C$. striatum of the upper respiratory tract with subsequent invasive infection. Therefore, $C$. striatum should not be simply disregarded as a contaminant, especially when isolated as a pure growth in chronically debilitated patients with multiple medical devices in situ (Brandenburg et al. 1996, Renom et al. 2007).

An increase in the number of cases in which C. striatum has been isolated from clinical specimens from patients with varying degrees of immunocompromisation and severe infections has been observed in industrialised countries that can afford these types of studies. With the aid of molecular-biology-based techniques, the clonal nature of the isolates in nosocomial outbreaks has been unequivocally established (Brandenburg et al. 1996, Martín et al. 2003, Otsuka et al. 2006, Renom et al. 2007, Adderson et al. 2008, Campanile et al. 2009, Martins et al. 2009, Wong et al. 2010).

In South America, only reports from Brazil indicate the sporadic isolation of $C$. striatum from representative clinical sites of hospitalised patients with signs and symptoms of infection (Camello et al. 2003, Martins et al. 2009, Superti et al. 2009). In contrast to previous data observed by our research group (Camello et al. 2003), an unusual clustering of 14 patients in our teaching hospital within a period of nine months produced a sentinel signal that justified the study of a possible outbreak. Therefore, the microbiologic characteristics, resistance profiles and similarities among the genomes of 15 C. striatum strains isolated from these patients were investigated. 


\section{SUBJECTS, MATERIALS AND METHODS}

Bacterial strains - We reviewed the microbiological features of 15 C. striatum strains recovered from representative clinical sites of 14 hospitalised patients $(50 \%$ male; $50 \%$ with fatal outcomes) with signs and symptoms of bacterial infection. The patients were hospitalised between August 2009-April 2010 in seven different wards of a 600-bed teaching hospital in Rio de Janeiro (RJ), Brazil (Table). All C. striatum isolates from the patients included in the study were detected using routine diagnostic cultures.

Bacterial phenotypic characterisation - Corynebacterium-like colonies were selected for further identification when they were grown in any quantity from normally sterile body fluid or when they were isolated in significant numbers or in pure culture from other specimens obtained at clinical sites where infection was suspected (Funke \& Bernard 2007). All clinical samples yielding more than three organisms were regarded as contaminated and discarded (Thomson \& Miller 2007). For quantitative bronchoalveolar lavage (BAL) fluid cultures, a colony count $>10^{3}$ colony-forming units (CFU) $\mathrm{mL}^{-1}$ of potential pathogens was considered positive. Isolation of three species of microorganisms was classified as a polymicrobial infection of the lower respiratory tract. Microorganisms were identified from the urine cultures in cystine lactose electrolyte-deficient agar (Merck, Darmstadt, Germany) and were considered potential pathogens when the growth exceeded $10^{4} \mathrm{CFU}$ $\mathrm{mL}^{-1}$ as the only isolate or $>10^{5} \mathrm{CFU} \mathrm{mL} \mathrm{m}^{-1}$ as the predominant isolate; in cases of nephropathies, $>10^{3} \mathrm{CFU}$ $\mathrm{mL}^{-1}$ was also considered a potential pathogenic level. Blood cultures were always obtained in pairs, wherein at least one of the samples was collected through the central venous catheter, if present. Blood specimens were inoculated in Bactec Plus anaerobic/aerobic vials and processed in a Bactec 9240 continuous-monitoring system (Becton-Dickinson Microbiology System, Cockeysville, MD, USA). Other clinical specimens were inoculated onto a Columbia agar base with the addition of $5 \%$ sheep's blood and incubated at $37^{\circ} \mathrm{C}$ in a $3-5 \%$ $\mathrm{CO}_{2}$ atmosphere and monitored for $72 \mathrm{~h}$ (Camello et al. 2003, Martins et al. 2009). Positive bacterial cultures for irregular Gram-positive rods were preliminarily characterised by colonial morphology, pigmentation, haemolysis, DNase activity and CAMP reaction with Staphylococcus aureus (Camello et al. 2003, Funke \& Bernard 2007, Pimenta et al. 2008). Phenotypic characterisation was also performed using the semi-automated API Coryne System 3.0 (bioMérieux) with the API web decoding system (apiweb.biomerieux.com) (Almuzara et al. 2006, Funke \& Bernard 2007, Campanile et al. 2009, Martins et al. 2009).

Susceptibility testing - Antimicrobial susceptibility profiles were determined by the disk diffusion method in cation-adjusted Mueller-Hinton agar supplemented with 5\% sheep blood. Breakpoints for the susceptible strains were used as suggested by the Clinical Laboratory Standards Institute (CLSI) for bacteria excluded from tables 2A-K. As there is not yet a defined standard for interpreting these results, the standard proposed in CLSI document M45-A (ISBN 1-56238-607-7) was used (CLSI 2007). The breakpoints for $S$. aureus were considered in the cases of penicillin, oxacillin and ampicillin. For the other antimicrobial agents, we used the breakpoints for other microorganisms, but not Haemophilus spp or Neisseria gonorrhoeae, which had been validated by previous studies. Intermediate results were considered resistant (Camello et al. 2003, Martins et al. 2009). The antibiotics (Oxoid SA, Spain) tested included penicillin $(10 \mathrm{U})$, ampicillin $(30 \mu \mathrm{g})$, methicillin $(5 \mu \mathrm{g})$, cefotaxime $(30 \mu \mathrm{g})$, cefepime $(30 \mu \mathrm{g})$, ceftriaxone $(30 \mu \mathrm{g})$, imipenem $(10 \mu \mathrm{g})$, erythromycin $(15 \mu \mathrm{g})$, clindamycin $(2 \mu \mathrm{g})$, linezolid (30 $\mu \mathrm{g})$, ciprofloxacin $(5 \mu \mathrm{g})$, moxifloxocin $(5 \mu \mathrm{g})$, tetracycline $(30 \mu \mathrm{g})$, gentamicin $(10 \mu \mathrm{g})$, rifampin $(5 \mu \mathrm{g})$, fosfomycin $(200 \mu \mathrm{g})$, vancomycin $(30 \mu \mathrm{g})$, mupirocin $(200 \mu \mathrm{g})$, tobramycin $(10 \mu \mathrm{g})$, nitrofurantoin $(300 \mu \mathrm{g})$ and ticarcillin/clavulanate $(75 \mu \mathrm{g} / 10 \mu \mathrm{g})$.

Gene amplification and sequencing - C. striatum identification was confirmed by $16 S$ rRNA and $r p o \mathrm{~B}$ gene sequencing. Each strain was grown in Brain Heart Infusion broth by incubation for $24 / 48 \mathrm{~h}$ at $30^{\circ} \mathrm{C}$ and centrifuged for $5 \mathrm{~min}$ at $3,000 \mathrm{rpm}$. The pelleted bacteria were suspended in $500 \mu \mathrm{L}$ of sterile water and subsequently boiled for 15 min for DNA extraction. Cell extracts were then immediately stored at $-20^{\circ} \mathrm{C}$ for use in polymerase chain reaction (PCR) reactions. $16 \mathrm{~S}$ $r R N A$ gene was amplified using universal primers $\mathrm{pA}$ (5'-AGAGTTTGATCCTGGCTCAG) and pH (5'-AAGGAGGTGATCCAGCCGCA), as described by Watts et al. (2000). The purified PCR product was sequenced by primer walking with the oligonucleotides using the following primers for sequencing: 1831 (5'-GAGGAACACCGATGGCGAAGGC), 1832 (5'-GCCCCCGTCAATTCCTTTGAGTT) (Watts et al. 2000) and 519r (5'G(AT)ATTACCGCGGC(GT)GCTG) and 1242f (5'CACACGTGCTACAATGG) (Johnson 1994). The rpoB gene was amplified and sequenced using primers following procedures described previously (Khamis et al. 2004). The sequencing reactions were performed using a BigDye Terminator v 3.1 cycle sequencing kit (Applied Biosystems) on an ABI-3730 Automated DNA Sequencer (Applied Biosystems) following standard protocols. The 16S rRNA gene sequences were compared to those available in the National Center for Biotechnology Information (ncbi.nlm.nih.gov) using the BLAST algorithm and the Ribosomal Database Project II (rdp.cme.msu. edu/html). The rpoB gene sequences were only compared in the GenBank database.

Phylogenetic analysis - The 16S rRNA gene sequences were aligned by CLUSTALX (Thompson et al. 1997). The phylogenetic trees were constructed by using the neighbour-joining (NJ) genetic distance method and were made using the program MEGA 4.0 package with the option of complete deletion of gaps (Tamura et al. 2007). The Kimura two-parameter model was chosen for all NJ tree constructions. The reliability of each tree topology was checked with 1,000 bootstrap replications. 


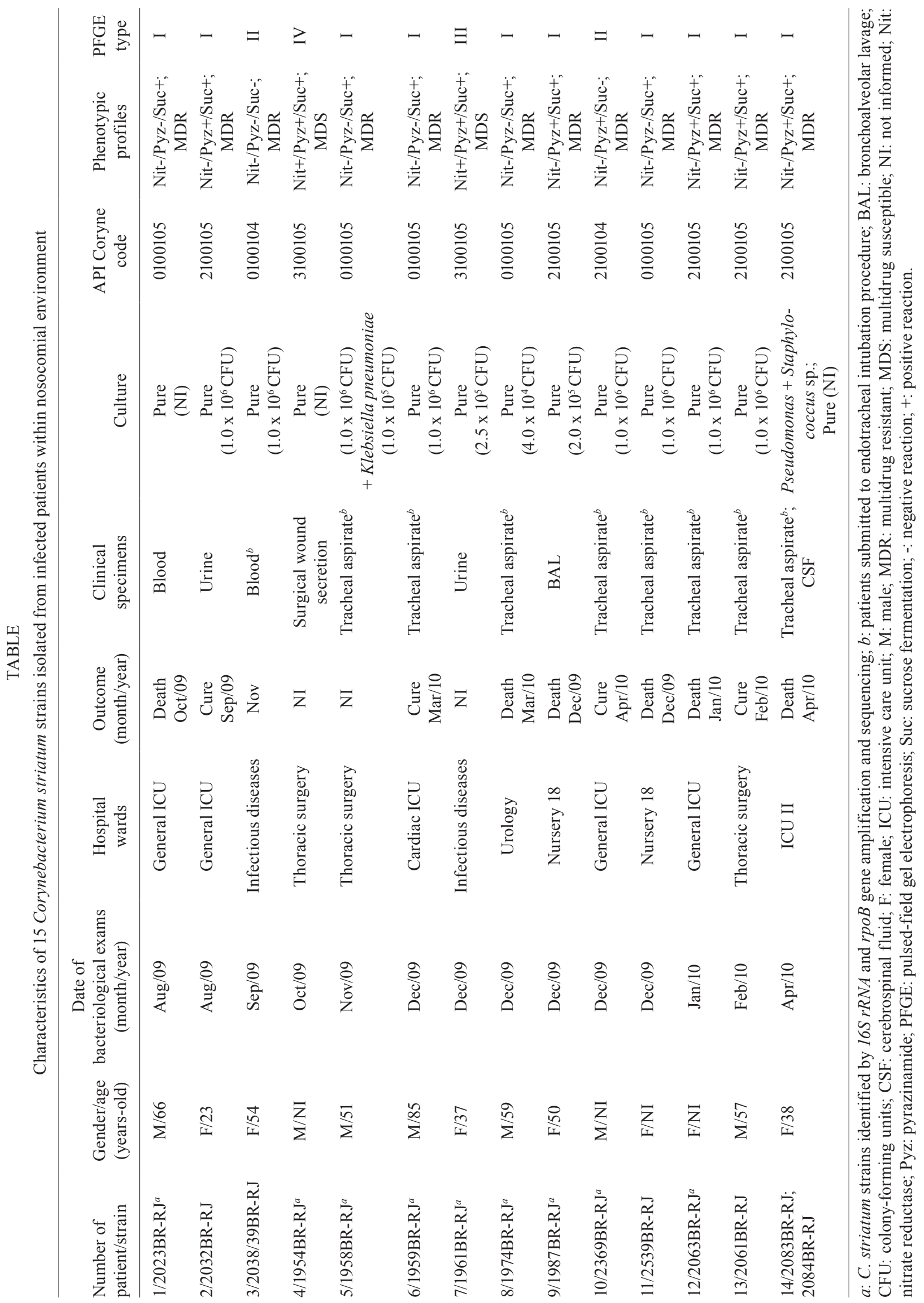


Pulsed-field gel electrophoresis (PFGE) - Genomic DNA was prepared following a method described previously (García-Crespo et al. 2005). The DNA was cleaved with SwaI (New England BioLabs) according to the manufacturer's instructions. PFGE was carried out in $0.5 \mathrm{X}$ TRIS-borate-EDTA- $1.2 \%$ agarose gels at $13^{\circ} \mathrm{C}$ with a CHEF DRII system (Bio-Rad). The pulse times were 1-30 $\mathrm{s}$ over $22 \mathrm{~h}$. A lambda DNA concatemer (New England BioLabs) was used as a molecular size marker. Similarities among macrorestriction patterns were identified according to the criteria established by Tenover et al. (1995).

Ethics - This study was developed in compliance with the Brazilian Government's Ethical Guidelines for research involving human beings (resolution of the National Health Council/Ministry of Health) and approved by the ethical research committee (INCA-CEP 008/06).

\section{RESULTS}

Epidemiological aspects - All 15 C. striatum clinical samples and the patients' ages, genders, hospital wards, outcomes, sites of isolation and associated microorganisms are displayed in chronological order in the Table. With the exception of four patients for whom age was not available, C. striatum strains were isolated only from adults ( 7 males and 7 females; half of whom were 50 years of age or older). Most of the strains were grown in pure culture $(n=13)$ and from tracheal aspirates $(n=9)$. Numerically predominant colonies of $C$. striatum were observed in two polymicrobial cultures from tracheal samples: one co-infected with Klebsiella pneumoniae and another with Pseudomonas sp. and Staphylococcus sp. C. striatum strains were also isolated from blood $(\mathrm{n}=1)$, cerebrospinal fluid $(\mathrm{CSF})(\mathrm{n}=1), \operatorname{BAL}(\mathrm{n}=1)$, surgical wounds $(\mathrm{n}=$ $2)$ and urine $(n=2)$ samples. From the deceased patient, number 14, C. striatum strains were isolated from both the tracheal aspirate and the CSF (Table).

When considering bacterial growth in pure culture and the proximity between microbiological diagnosis and death of the patients, the possibility of a causal link between death and $C$. striatum infection was observed in five cases (3, 9, 11, 12 and 14) (Table). At least half of the patients previously infected with C. striatum died, indicating a high severity of illness and/or immune deficiency. All seven deaths during the period of study occurred in patients from whom C. striatum was isolated in a pure culture (3 in December 2009).

Fig. 1 presents the epidemic curve of the infections caused by $C$. striatum, suggestive of a nosocomial outbreak. Most of the C. striatum strains $(\mathrm{n}=6)$ were isolated in December 2009 from patients from various hospital wards. The pathogen was found circulating mostly among inpatients admitted to the ICU and surgical wards $(\mathrm{n}=10)$ from August 2009-April 2010 (Table). C. striatum infections were observed in the general ICU $(\mathrm{n}=4)$, cardiac ICU $(\mathrm{n}=1)$, ICU II $(\mathrm{n}=2)$, thoracic surgery ( $=3)$, nursery $18(\mathrm{n}=2)$, infectious diseases $(\mathrm{n}=2)$ and urology $(\mathrm{n}=1)$ wards.

Antimicrobial susceptibility, phenotypic and genotypic properties of C. striatum clinical isolates - MDR profiles for 21 of the antimicrobial agents tested were

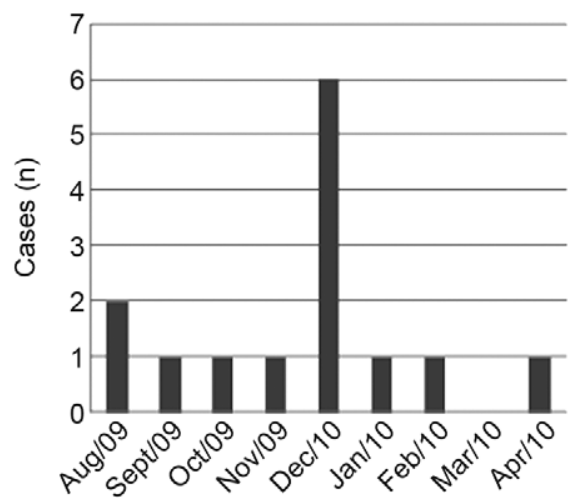

Fig. 1: epidemic curve for Corynebacterium striatum over time.

observed in $87 \%$ of the $C$. striatum strains that were susceptible only to vancomycin, linezolid and tetracycline. Two strains (1954BR-RJ surgical wound isolate and 1961BR-RJ urine isolate) were susceptible to most of the tested drugs (MDS) except mupirocin, fosfomycin and ticarcillin/clavulanate. Deaths occurred only in patients from whom MDR C. striatum strains were isolated in the blood, CFS, BAL and/or tracheal aspirate.

The phenotypic analysis of $C$. striatum strains revealed variability in the results of nitrate reductase and pyrazinamide (pyz) activities and/or sucrose fermentation (Table). To genotypically confirm the suspected $C$. striatum isolates, we performed $16 \mathrm{~S}$ rRNA and rpoB gene sequencing. GenBank accessions for the 16S rRNA and rpoB genes sequences were deposited: JF342692-JF342700 and JF342701-JF342709, respectively. The 16S rRNA sequences from the clinical isolates exhibited the highest similarity values to the type strain of $C$. striatum (ATCC 6940), ranging from $99.32-99.84 \%$. The phylogenetic analysis, based on the 16S rRNA sequences, unambiguously demonstrated that the clinical isolates belonged to the $C$. striatum species, as illustrated in Fig. 2. A comparative analysis of the partial $r p o \mathrm{~B}$ sequences for the clinical isolates revealed sequence similarities in the ranges of 97.08$97.15 \%$ with the type strain C. striatum (ATCC 6940).

PFGE analysis - The restriction endonuclease SwaI revealed four distinct PFGE profiles among the C. striatum isolates, which were designated I, II, III and IV. The PFGE profile I was the most frequently observed among the 15 strains $(78.57 \%)$ isolated from the 14 patients (71.42\%) included in this study (Fig. 3). PFGE analysis confirmed the isolation of a single clone of $C$. striatum (PFGE I) from the respiratory tract and CSF of the patient of case 14. Profiles II, III and IV differed by more than three bands from PFGE profile I by visual inspection. According to the interpretation criteria by Tenover et al. (1995), MDR clinical isolates belonging to profile II exhibited six bands of difference from the outbreak MDR strain and were considered potentially related to the outbreak. The MDS isolates belonging to profiles III and IV exhibited more than six bands of difference, indicating that they were unrelated to the outbreak strain (Fig. 3). 


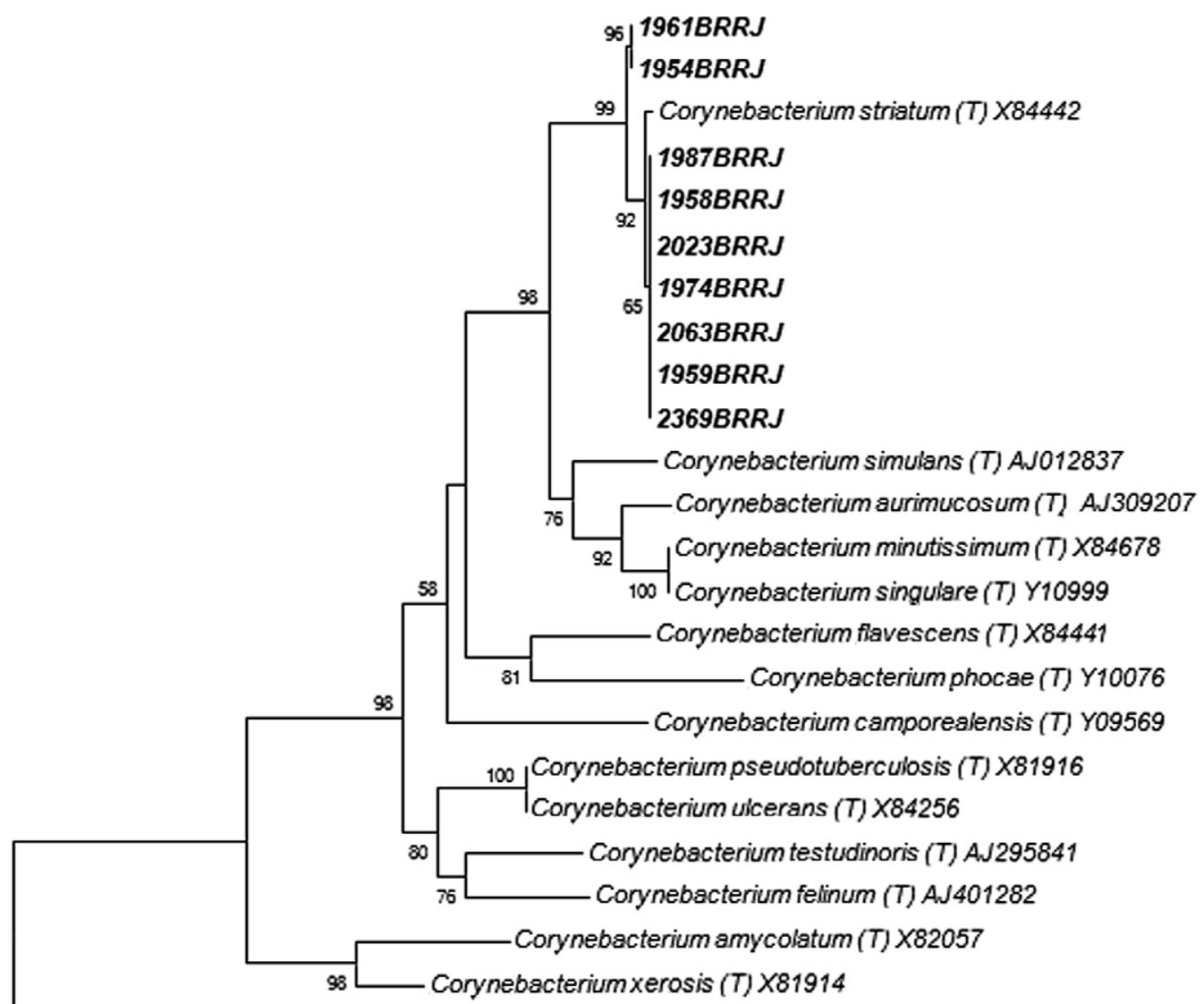

Actinomyces bovis $(T) \times 81061$

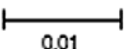

Fig. 2: phylogenetic tree based on neighbour-joining method using $16 \mathrm{~S} r R N A$ gene sequences. Distance estimations were calculated by using the Kimura two-parameter model. Bootstrap percentages after 1,000 simulations are shown. The Actinomyces bovis (T) X81061 sequence was used as outgroup.

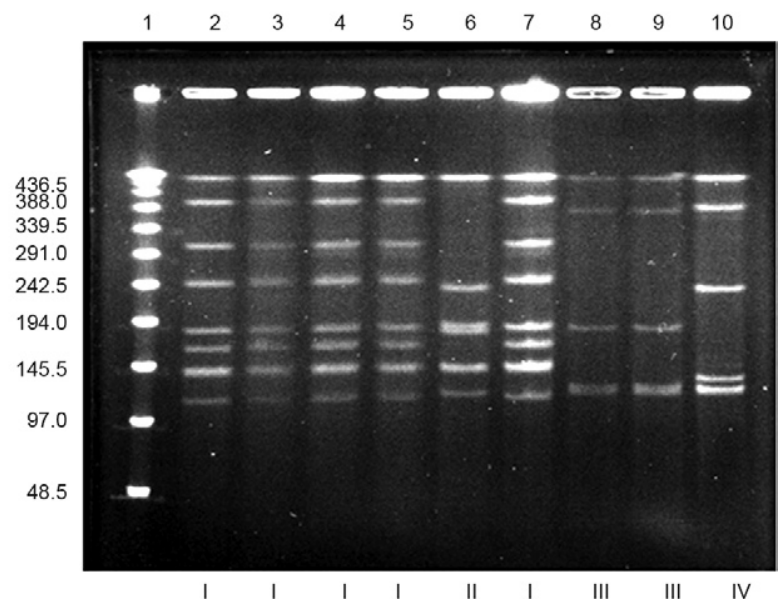

Fig. 3: pulsed-field gel electrophoresis (PFGE) profiles of Brazilian Corynebacterium striatum isolates. Lane 1: $\lambda$ DNA ladder PFGE marker; 2-5, 7: profile I (isolates 1958, 1959, 1987, 1974 and 2539, respectively); 6: profile II (isolate 2369); 8, 9: profile III (isolate 1961); 10: profile IV (isolate 1954).

\section{DISCUSSION}

In Brazil, previous investigations revealed that 1.9\% of samples isolated from varied clinical sources of cancer patients treated in a reference centre in RJ during a one-year period were positive for C. striatum, corresponding to one case of upper and three of lower respiratory tract infections and two cases of surgical wounds (Martins et al. 2009).

In the present study, we documented a nosocomial outbreak that includes a fatal case of systemic infection caused by $C$. striatum in a 600-bed teaching hospital in RJ. PFGE analyses of 15 C. striatum strains indicated the presence of four PFGE profiles, including two related clones of MDR strains (PFGE I and II). Our data demonstrated the predominance of PFGE type I comprising $11 \mathrm{MDR}$ isolates that were mostly isolated from the ICU and surgical wards. The tracheal aspirate specimens obtained from patients with ventilator-associated respiratory tract colonisation or pneumonia were mostly (6 out of 7) PFGE type I. The possibility of a causal link between death and C. striatum (PFGE types I and II) 
infection was observed on five occasions. Similar to our findings, previous studies also revealed ventilator support as a relevant risk factor for acquiring C. striatum infection (Brandenburg et al. 1996, Campanile et al. 2009, Wong et al. 2010).

Investigations dealing with infections caused by coryneform bacteria in paediatric oncology patients of St. Jude Children's Research Hospital (Tennessee, USA) indicated that most bacteraemia cases were due to C. striatum. These patients had complications related to their infections, including infection relapse and septic arthritis (Adderson et al. 2008). In the present study, C. striatum strains were not isolated from infants and new-borns and/or cancer patients. The pathogen was isolated from adults, with half of the patients $(\mathrm{n}=7) 50$ years of age or older. The isolation of pure, heavy-growth C. striatum in the absence of other pathogens together with clinical deterioration provided strong evidence for its pathogenic role in our patients. However, we could not ascertain how many of the fatalities could be attributed to C. striatum infection rather than to underlying conditions.

Except for the unvarying activity of vancomycin against corynebacteria, the variability in resistance to other classes of antimicrobial agents emphasises the need for the continuous surveillance of their resistance patterns. Although most reported C. striatum isolates have been susceptible to a wide range of antibiotics (Martínez-Martínez et al. 1995, 1996, Weiss et al. 1996), it has been suggested that the selective pressure exerted by prior antimicrobial treatment favours the overgrowth of $C$. striatum as a secondary coloniser in immunocompromised hosts. In this context, the emergence of MDR strains is of particular concern (Leonard et al. 1994, Campanile et al. 2009). Herein, the MDR phenotype of $C$. striatum strains was immediately observed and was responsible for the alarm that led to the subsequent laboratory surveillance of these strains. Most (87\%) $C$. striatum strains were only susceptible to vancomycin, linezolid and tetracycline.

In Japan, Otsuka et al. (2006) reported variable rates of the susceptibility of $C$. striatum to $\beta$-lactams and aminoglycosides, with high levels of resistance to erythromycin, tetracycline, rifampin and ciprofloxacin, although all strains were sensitive to vancomycin. PFGE procedures identified 14 patterns of $C$. striatum, with types A, D and $\mathrm{E}$ associated with nosocomial outbreaks of respiratory origin and with subtypes A1, A2, D2 and $\mathrm{E}$ associated with resistance to a broad range of antibiotics. Moreover, Renom et al. (2007) observed in their samples that the criterion of multidrug resistance (resistance to 3 or more antibiotics of different families) applied to $100 \%$ of the strains isolated in nosocomial outbreaks, of which $65 \%$ were resistant to four or five different antibiotic groups, $6.9 \%$ were sensitive only to imipenem and vancomycin and $11 \%$ were sensitive only to vancomycin. According to the sensitivity patterns obtained by Otsuka et al. (2006), we observed in our samples that the criterion of multidrug resistance (resistance to 3 or more antibiotics of different families) applies to $87 \%$ of C. striatum (PFGE types I and II) strains isolated in the present nosocomial outbreak.
For C. striatum, no publically available database exists, such as PulseNet (cdc.gov/pulsenet), to enable the comparison of PFGE patterns observed in the different nosocomial outbreaks. Campanile et al. (2009) observed that the SwaI-PFGE profiles of C. striatum exhibit bands ranging in size from 48.5-533.5 kb. In our study, we obtained SwaI-PFGE profiles with bands ranging in size from over 97.0-533.5 kb. The absence of bands ranging in size from 48.5-97.0 kb suggested that the MDR $C$. striatum strains isolated in this nosocomial outbreak in Brazil were different from those isolated in Italy by Campanile et al. (2009). Moreover, the analysis of the phenotypic profiles of C. striatum indicated that the Brazilian strains were different from those isolated in the Netherlands (biotype: nitrate/pyz-positive and sucrosenegative; API code 3100104) (Brandenburg et al. 1996).

To our knowledge, this is the first Brazilian nosocomial outbreak caused by MDR C. striatum described in the literature. With the support of PFGE techniques, the clonal nature of the outbreak isolates was established, although a common source and the mode of transmission could not be determined. The present findings also highlight the importance of C. striatum as an emerging MDR nosocomial pathogen worldwide and the fact that different clones may be responsible for these nosocomial outbreaks.

\section{REFERENCES}

Adderson EE, Boudreaux JW, Hayden RT 2008. Infections caused by coryneform bacteria in pediatric oncology patients. Pediatr Infect Dis J 27: 136-141.

Almuzara MN, De Mier C, Rodríguez CR, Famiglietti AM, Vay CA 2006. Evaluation of API Coryne System version 2.0 for diphtheroid gram-positive rods identification with clinical relevance. Rev Argent Microbiol 38: 197-201.

Boltin D, Katzir M, Bugoslavsky V, Yalashvili I, Brosh-Nissimov T, Fried M, Elkayam O 2009. Corynebacterium striatum - a classic pathogen eluding diagnosis. Eur J Intern Med 20: 49-52.

Brandenburg AH, van Belkum A, van Pelt C, Bruining HA, Mouton JW, Verbrugh HA 1996. Patient-to-patient spread of a single strain of Corynebacterium striatum causing infections in a surgical intensive care unit. J Clin Microbiol 34: 2089-2094.

Camello TCF, Mattos-Guaraldi AL, Formiga LCD, Marques EA 2003. Nondiphtherial Corynebacterium species isolated from clinical specimens of patients in a University hospital, Rio de Janeiro, Brazil. Braz J Microbiol 34: 39-44.

Campanile F, Carretto E, Barbarini D, Grigis A, Falcone M, Goglio A, Venditti M, Stefani S 2009. Clonal multidrug-resistant Corynebacterium striatum strains, Italy. Emerg Infect Dis 15: 75-78.

CLSI - Clinical Laboratory Standards Institute 2007. Methods for antimicrobial dilution and disk susceptibility testing of infrequently isolated or fastidious bacteria. M45-A, CLSI, Wayne, $15 \mathrm{pp}$.

Fernández-Ayala M, Nan DN, Fariñas MC 2001. Vertebral osteomyelitis due to Corynebacterium striatum. Am J Med 111: 167.

Funke G, Bernard KA 2007. Coryneform gram-positive rods. In PR Murray, EJ Baron, JH Jorgensen, ML Landry, MA Pfaller, Manual of clinical microbiology, 9th ed., ASM Press, Washington DC, p. 485-514.

García-Crespo D, Navas J, Gradillas G, Juste RA 2005. Technical note: molecular typing of Corynebacterium bovis isolates by pulsed-field gel electrophoresis. J Dairy Sci 88: 1705-1707. 
Heidemann DG, Dunn SP, Diskin JA, Aiken TB 1991. Corynebacterium striatum keratitis. Cornea 10: 81-82.

Johnson JL 1994. Similarity analysis of rRNAs. In P Gerhardt, RGE Murray, WA Wood, NR Krieg, Methods for general and molecular bacteriology, American Society for Microbiology, Washington DC, p. 691.

Khamis A, Raoult D, La Scola B 2004. rpoB gene sequencing for identification of Corynebacterium species. J Clin Microbiol 42: 3925-3931.

Lee PP, Ferguson Jr DA, Sarubbi FA 2005. Corynebacterium striatum: an underappreciated community and nosocomial pathogen. $J$ Infect 50: 338-343.

Leonard RB, Nowowiejski DJ, Warren JJ, Finn DJ, Coyle MB 1994. Molecular evidence of person-to-person transmission of a pigmented strain of Corynebacterium striatum in intensive care units. J Clin Microbiol 32: 164-169.

López AB, Gil Ruiz MT, Vega PL, Fajardo OM 2009. Cystitis and haematuria due to Corynebacterium striatum. A case report and review. Actas Urol Esp 33: 909-912.

Martín MC, Melon O, Celada MM, Alvarez J, Mendez FJ, Vazquez F 2003. Septicaemia due to Corynebacterium striatum: molecular confirmation of entry via the skin. J Med Microbiol 52: 599-602.

Martínez-Martínez L, Pascual A, Bernard K, Suárez AL 1996. Antimicrobial susceptibility pattern of Corynebacterium striatum. Antimicrob Agents Chemother 40: 2671-2672.

Martínez-Martínez L, Suárez AI, Winstanley J, Ortega MC, Bernard K 1995. Phenotypic characteristics of 31 strains of Corynebacterium striatum isolated from clinical samples. J Clin Microbiol 33: $2458-2461$.

Martins CAS, Faria LMD, Souza MC, Camello TCF, Velasco E, Hirata Jr R, Thuler LCS, Mattos-Guaraldi AL 2009. Microbiological and host features associated with corynebacteriosis in cancer patients: a five-year study. Mem Inst Oswaldo Cruz 104: 905-913.

Moore K, Hall V, Paul A, Morris T, Brown S, McCulloch D, Richardson MC, Harding KG 2010. Surface bacteriology of venous leg ulcers and healing outcome. J Clin Pathol 63: 830-834.

Oliva A, Belvisi V, Iannetta M, Andreoni C, Mascellino MT, Lichtner M, Vullo V, Mastroianni CM 2010. Pacemaker lead endocarditis due to multidrug-resistant Corynebacterium striatum detected with sonication of the device. J Clin Microbiol 48: 4669-4671.
Otsuka Y, Ohkusu K, Kawamura Y, Baba S, Ezaki T, Kimura S 2006. Emergence of multidrug-resistant Corynebacterium striatum as a nosocomial pathogen in long-term hospitalized patients with underlying diseases. Diagn Microbiol Infect Dis 54: 109-114.

Pimenta FP, Souza MC, Pereira GA, Hirata Jr R, Camello TC, MattosGuaraldi AL 2008. DNase test as a novel approach for the routine screening of Corynebacterium diphtheriae. Lett Appl Microbiol 46: 307-311.

Renom F, Garau M, Rubi M, Ramis F, Galmes A, Soriano JB 2007. Nosocomial outbreak of Corynebacterium striatum infection in patients with chronic obstructive pulmonary disease. J Clin Microbiol 45: 2064-2067.

Scholle D 2007. A spontaneous joint infection with Corynebacterium striatum. J Clin Microbiol 45: 656-658.

Superti SV, Martins DS, Caierao J, Soares F, Prochnow T, Cantarelli VV, Zavascki AP 2009. Corynebacterium striatum infecting a malignant cutaneous lesion: the emergence of an opportunistic pathogen. Rev Inst Med Trop Sao Paulo 51: 115-116.

Tamura K, Dudley J, Nei M, Kumar S 2007. MEGA4: Molecular Evolutionary Genetics Analysis (MEGA) software version 4.0. Mol Biol Evol 24: 1596-1599.

Tenover FC, Arbeit RD, Goering RV, Mickelsen PA, Murray BE, Persing DH, Swaminathan B 1995. Interpreting chromosomal DNA restriction patterns produced by pulsed-field gel electrophoresis: criteria for bacterial strain typing. J Clin Microbiol 33: 2233-2239.

Thompson JD, Gibson TJ, Plewniak F, Jeanmougin F, Higgins DG 1997. The CLUSTALX windows interface: flexible strategies for multiple sequence alignment aided by quality analysis tools. Nucleic Acids Res 25: 4876-4882.

Thomson Jr RB, Miller M 2007. Specimen collection, transport and processing: bacteriology. In PR Murray, EJ Baron, JH Jorgensen, ML Landry, MA Pfaller, Manual of clinical microbiology, ASM Press, Washington DC, p. 286-330.

Watts JL, Lowery DE, Teel JF, Rossbach S 2000. Identification of Corynebacterium bovis and other coryneforms isolated from bovine mammary glands. J Dairy Sci 83: 2373-2379.

Weiss K, Labbé AC, Laverdière M 1996. Corynebacterium striatum meningitis: case report and review of an increasingly important Corynebacterium species. Clin Infect Dis 23: 1246-1248.

Wong KY, Chan YC, Wong CY 2010. Corynebacterium striatum as an emerging pathogen. $J$ Hosp Infect 76: 371-372. 